\title{
Training-Aided Coherent Optical Single-Carrier System With Improved Nonlinearity Tolerance
}

Chen Zhu, An V. Tran, Senior Member, IEEE, Liang B. Du, Member, IEEE, and Arthur J. Lowery, Fellow, IEEE

\begin{abstract}
In this letter, we propose a nonlinearity-tolerant channel estimation technique for training-aided singlecarrier polarization-division-multiplexed (PDM) coherent optical systems. Simply by compensating the effects of fiber nonlinearity on the received training sequence, linear channel estimation and equalization can be improved; this approach is very efficient, which requires a negligible computational effort since training only occupies a very small portion of the total data. The concept is verified using simulations for both QPSK and 64-quadrature amplitude modulation (QAM) systems and experimental measurements for a 120-Gb/s PDM 64-QAM system with 800-km erbium-doped fiber amplifier (EDFA)-only transmission. The experimental results show that the system performance is improved by $0.4 \mathrm{~dB}$ at the optimal launched power and $1.6 \mathrm{~dB}$ in the highly nonlinear region, compared with conventional training-aided channel estimation approaches.
\end{abstract}

Index Terms-Coherent optical system, 64-QAM, MIMO equalization, training sequence, nonlinearity compensation.

\section{INTRODUCTION}

$\mathbf{C}$ OHERENT detection in combination with digital signal processing (DSP) has been demonstrated as the most promising technique to realize high-capacity optical transmission networks [1]. Polarization-division-multiplexing (PDM) with high-level quadrature amplitude modulation (QAM) formats is being used to implement high spectral efficiency (SE) coherent optical transmission. Recently, intensive research on single-carrier PDM $m$-QAM $(m>4)$ systems has achieved high SE [2]-[6]. In most previous works, multipleinput multiple-output (MIMO) adaptive equalization is used for polarization de-multiplexing (pol-demux). To address the multi-modulus feature of $m$-QAM formats $(m>4)$, multi-stage MIMO adaptive filters are required, which raises the computational effort and system latency compared with lower-order modulation formats such as QPSK. Moreover, the carrier recovery may be needed before the adaptive filter switches to decision-directed steady state optimization, where

Manuscript received February 19, 2014; revised March 25, 2014; accepted April 14, 2014. Date of publication April 24, 2014; date of current version May 16, 2014.

C. Zhu is with the National ICT Australia, Victoria Research Laboratory, Department of Electrical and Electronic Engineering, University of Melbourne, Parkville, VIC 3000, Australia (e-mail: zhuc@student.unimelb.edu.au).

A. V. Tran is with the Centre for Technology Infusion, La Trobe University, Bundoora, VIC 3010, Australia (e-mail: tranav@unimelb.edu.au).

L. B. Du is with Google, Mountain View, CA 94043 USA (e-mail: liangbydu@gmail.com).

A. J. Lowery is with the Centre for Ultrahigh Bandwidth Devices for Optical Systems, Department of Electrical and Computer Systems Engineering, Monash University, Clayton, VIC 3800, Australia (e-mail: arthur.lowery@monash.edu).

Color versions of one or more of the figures in this letter are available online at http://ieeexplore.ieee.org.

Digital Object Identifier 10.1109/LPT.2014.2319235 frequency and phase estimation errors due to imperfect filter pre-convergence may then cause performance penalty.

Training-aided channel estimation (TA-CE) is another reliable solution for pol-demux; by sending the training sequence (TS) periodically, feed-forward and modulation format-flexible channel estimation (CE) can be attained and updated [5]-[8]. Furthermore, TA-CE is always combined with a one-tap frequency domain equalizer to realize computationally efficient data recovery. Compared with blind adaptive schemes, a greater benefit can be attained with higher-order modulation formats when using TA-CE, since the same channel estimation and equalization structure is maintained for TA-CE systems as with the low-order modulation format. However, a PDM $m$-QAM system with $m>16$ using TA-CE has yet to be demonstrated experimentally. Furthermore, the performance of TA-CE has not been investigated in the presence of fiber nonlinearity, which becomes another motivation for this work.

In this letter, we first show that TA-CE has a much poorer nonlinearity-limited performance than a blind adaptive scheme. Then, we propose a nonlinearity tolerant TA-CE (NLT-TA-CE) method based on digital backpropagation (DBP) [10] of just the TS. This approach only requires a very small increase of computational power since the TS is typically a very small fraction of the total data sent. We use numerical simulations and 800-km 10-Gbaud PDM 64-QAM experiments to verify the concept, and show that NLT-TA-CE improves the received signal $Q^{2}$ factor by $0.4 \mathrm{~dB}$ at the optimal launched power and by up to $1.6 \mathrm{~dB}$ in the highly-nonlinear region. This letter is an extension of the original work [9], where a number of significantly new contents have been added: the theoretical analysis of the training-aided system in the presence of fiber nonlinearity in Section II; the 64-QAM simulation results and discussions that appear in Section III; the blind equalization results of 64-QAM experiment in Section IV.

\section{NONLINEARITY-TOLERANT TRAINING-AIDED FREQUENCY-DOMAIN EQUALIZATION}

For a coherent optical single-carrier system with only linear equalization, the performance penalty at higher powers is due to two factors: $(i)$ intra-channel fiber nonlinearity degrades the payload signal, (ii) nonlinearity degrades the linear CE, which leads to imperfect linear equalization. Although $(i)$ is the dominant factor and could be mitigated using digital nonlinearity compensation [10], [11], its high computational complexity prevents real-time implementation. Therefore, it is prudent to consider optimizing factor (ii) independently.

With blind adaptive pol-demux, the MIMO filter converges to an accurate linear solution under both ASE-limited and 


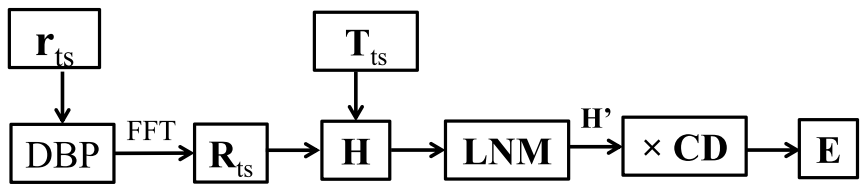

Fig. 1. Receiver structure for NLT-TA-CE. DBP: digital backpropagation, LNM: linear noise removal.

nonlinearity-limited cases. While with normal TA-CE, the CE is derived from the received TS, which can be modeled as:

$$
\mathbf{R}_{T S}=\mathbf{H} \cdot \mathbf{T}_{T S}+\mathbf{L N}
$$

where all notations are in frequency domain: $\mathbf{R}_{T S}$ denotes the received training matrix, $\mathbf{H}$ is the $2 \times 2$ linear MIMO channel, $\mathbf{T}_{T S}$ is the transmitted training matrix and $\mathbf{L N}$ indicates the linear noise, which is the combination of ASE noise and electronic noise including shot noise, electrical thermal noise and quantization noise. Various TS algorithms have been developed to estimate the channel [5]-[8], which find the estimated channel $\mathbf{H}^{\prime}=\mathbf{R}_{T S} / \mathbf{T}_{T S}$. Then the effect of noise is suppressed by either applying boxcar averaging over several received TSs [7], or by transforming back to time-domain to drop the non-effective taps for noise removal [5]. These methods work well in the linear region.

In the presence of non-negligible fiber nonlinearity, Equation (1) may be rewritten as:

$$
\mathbf{R}_{T S}=\mathbf{H} \cdot \mathbf{T}_{T S}+\mathbf{L N}+\mathbf{N L I}
$$

where the nonlinear interference (NLI) is modeled as an additive term, which is uncorrelated with the linear noise and the signal. Although the NLI can be described using Gaussian noise analysis [12], it is data-pattern dependent, i.e. the selfphase modulation within identical signal blocks, such as the training blocks, would be the same. Therefore the effect of NLI is preserved upon averaging identical TS blocks.

The proposed NLT-TA-CE structure is illustrated in Fig. 1. The received time-domain TS $\left(\mathbf{r}_{t s}\right)$ first passes through a DBP module (TS-DBP) for nonlinearity compensation, then is transformed into frequency domain $\left(\mathbf{R}_{t s}\right)$ using Fast Fourier transforms (FFT), and then conventional TA-CE estimates the $2 \times 2$ MIMO PDM channel $\mathbf{H}$ [7]. The linear noise is reduced using time-domain windowing [5]. The data payload is not processed by DBP. The NLT-TA-CE is obtained after combining $\mathbf{H}$ ' with the $\mathrm{CD}$ transfer function (i.e. multiplying the $\mathrm{CD}$ transfer function with each of the four components of the $2 \times 2$ channel matrix linearly). Finally, the MIMO frequency domain equalizer coefficients $\mathbf{E}$ are set according to MMSE criterion, which is able to compensate for all of the linear channel impairments using a single linear filter.

It is worth noting that the NLT-TA-CE is independent of the employed TS. NLT-TA-CE can be applied if the durations of the TSs are able to cover the whole channel memory (mainly CD memory). Or in other words, NLT-TA-CE is not applicable for systems with electronic $\mathrm{CD}$ equalization and for those using a short TS designed to only aid pol-demux [6], since the TS is too short to apply DBP in this case.
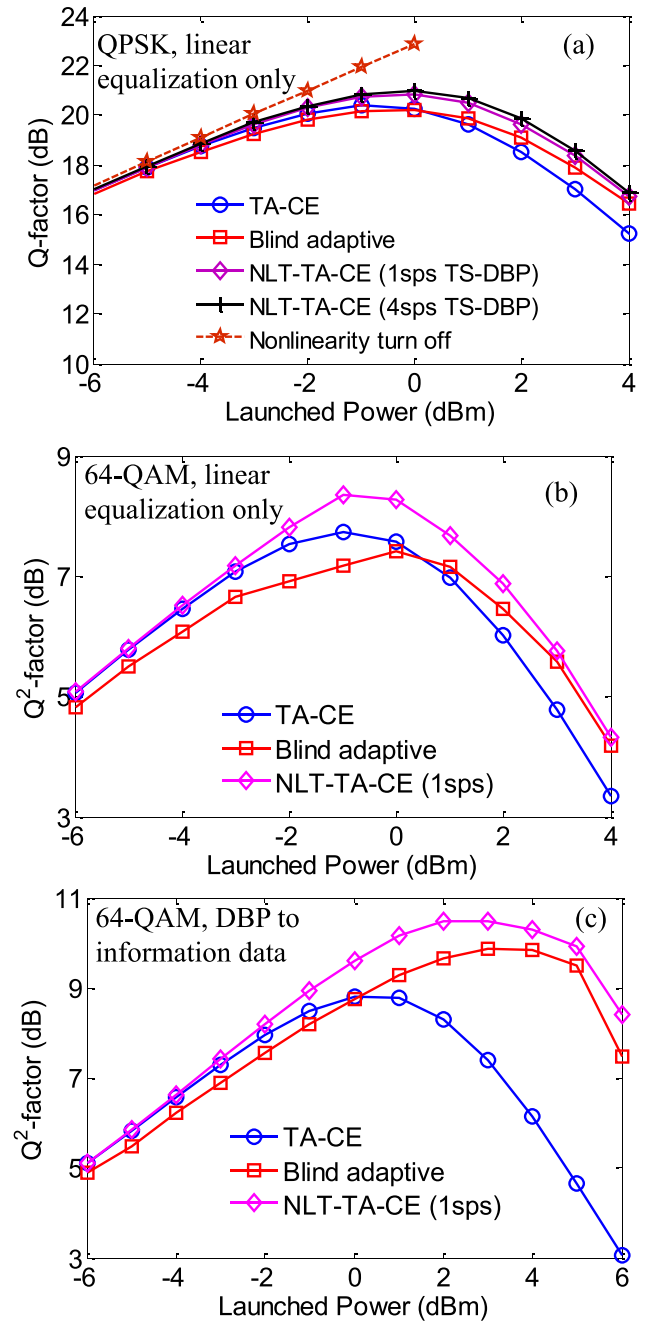

Fig. 2. Simulation results with linear equalization only for (a) QPSK and (b) 64-QAM systems, and (c) 1sps DBP applied to information symbols.

\section{Simulation Results}

The optimality of linear equalizers in the presence of intra-channel fiber nonlinearity was examined in a 20-GBaud single channel PDM simulation using VPItransmissionMaker 8.7, with the following parameters: $100-\mathrm{kHz}$ linewidth for both transmitter laser and local oscillator, $\pm 1 \mathrm{GHz}$ frequency offset, 800-km EDFA-only transmission link with $80-\mathrm{km}$ spans, $0.2-\mathrm{dB} / \mathrm{km}$ fiber loss, $2.6 \times 10^{-20} \mathrm{~m}^{2} / \mathrm{W}$ nonlinearity coefficient, 5-ps/ $/ \mathrm{km}^{1 / 2}$ PMD coefficient and 6-dB EDFA noise figure. For the blind equalization systems, after standard overlap-add CD equalization, constant modulus algorithm (CMA) [13] was applied for QPSK. While for 64-QAM, the adaptive equalizer was pre-converged using CMA plus multi-modulus algorithm (MMA) [14]. Then the blind adaptation was conducted with joint carrier recovery and decisiondirected least mean square (DD-LMS) [15]. It is clear that for blind equalization, the filter setup becomes more complicated as the modulation format order increases. On the other hand, a Golay sequence [7] was used as the TS for training-aided systems, and the CE and equalizer setup was the same for both modulation formats.

Figure 2 shows the results for both QPSK and 64-QAM simulations. For QPSK system since some cases had no bit errors, 
the $Q$-factors which derived from the constellation variance were used for signal quality evaluation. While for 64-QAM system the $Q^{2}$-factor was calculated based on bit error rate $(\mathrm{BER})$ using $Q^{2}(d B)=20 \log _{10}\left(\sqrt{2} e r f c^{-1}(2 B E R)\right)$. The performance of different linear equalization systems for QPSK is plotted in Fig. 2(a) TA-CE performs slightly better than blind adaptive equalization in the linear region (from -6 to $0 \mathrm{dBm}$ ), this is mainly due to the limited number of samples used for convergence and the carrier recovery error propagates to the decision-directed adaptation stage. The blind equalizer achieves better linear channel acquisition than TA-CE when the launched power is beyond the nonlinear threshold. This is because the linear CE of TA$\mathrm{CE}$, becomes inaccurate due to NLI that distorts the TS. This is worse than the convergence error of the adaptive algorithm due to fiber nonlinearity. The NLT-TA-CE with 1 step-perspan (sps) TS-DBP offers about 0.5-dB $Q$-improvement at the optimal launched power compared with the normal TA-CE, making its performance similar to the blind adaptive scheme within the highly nonlinear region. Its performance in the linear region is slightly enhanced $(0.1$ to $0.2 \mathrm{~dB})$ because the linear $\mathrm{CE}$ is also improved even with very weak nonlinearities. Increasing the number of steps from $1 \mathrm{sps}$ to 4 sps for TS-DBP can only improve the performance slightly, since the performance is still limited by the nonlinear distortion of the information symbols. Thus, we used 1 sps TS-DBP for the remainder of the letter, which minimizes the computational complexity. Since the TS is only a small proportion of the total signal duration (e.g. 1.96\% in [5]), the additional TS-DBP process adds only a negligible computational burden to the total system. Figure 2(b) shows the performance of three 64-QAM systems using linear equalization only, and it is similar to the QPSK results in Fig. 2(a), except that the adaptigve equalizer performs slightly worse in linear region than TA-CE systems.

To illustrate the performance difference between these two linear channel equalizers more clearly, DBP with $1 \mathrm{sps}$ was applied to the information symbols before pol-demux with 64-QAM format. Fig. 2(c) shows that the adaptive channel acquisition is also improved by using DBP on the payload; the blind equalizer outperforms the conventional TA-CE (where $\mathrm{CD}$ is removed from TA-CE) in the nonlinear regions with about 1.5 -dB higher peak $Q$-performance. This difference is purely due to the difference between the optimal and the nonlinearity distorted linear channel estimations, confirming the validity of factor (ii) described in Section II. When applying NLT-TA-CE and DBP to the data payload, the performance catches up with the blind adaptive scheme. Simulation results verified that the fiber-nonlinearity limited performance of training-aided system can be enhanced by using NLT-TA-CE, and in highly nonlinear region NLT-TA-CE system also achieves comparable performance to the blind scheme, while maintaining the benefits such as modulationformat flexibility and low implementation-complexity.

\section{PDM-64QAM EXPERIMENTAL DEMONSTRATION}

Figure 3(a) shows the block diagram of the experimental setup. TA-CE, blind equalization and NLT-TA-CE
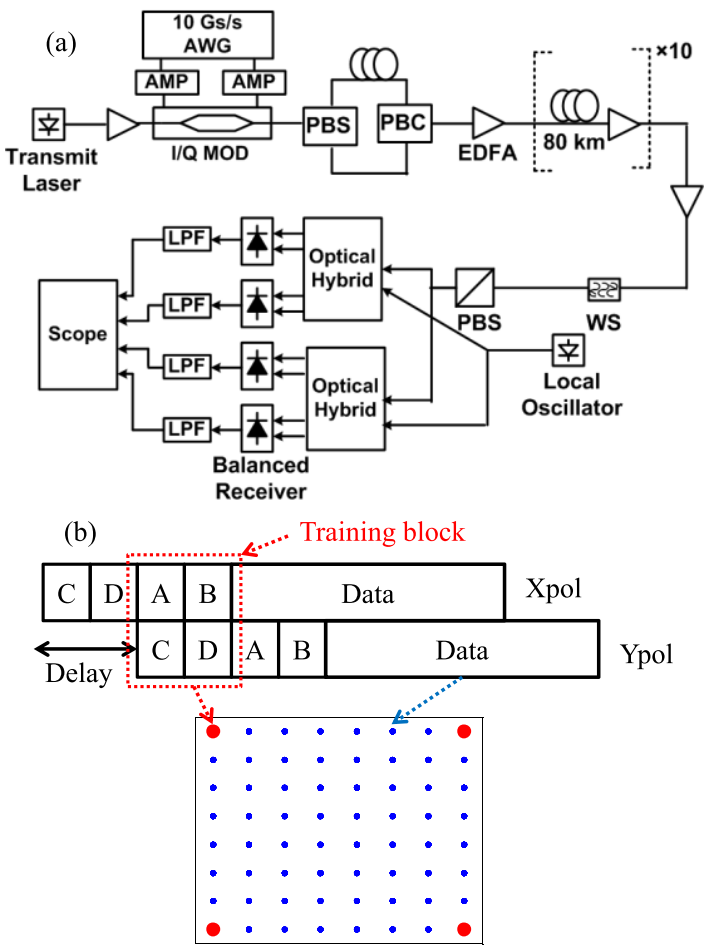

Fig. 3. (a) Experimental setup, (b) transmitted signal structure. AMP: RF amplifier, I/Q MOD: I/Q modulator.

were compared. A 10-Gsamples/s arbitrary waveform generator (AWG) generated baseband signals that drive an optical I/Q modulator. The output of the transmitter laser was then modulated to provide the optical signals. PDM was emulated by splitting the optical signals into two paths with a polarization beam splitter (PBS), where one path experienced 19,600-ps delay relative to the other. The two tributaries were recombined through a polarization beam combiner (PBC). The transmitted PDM signal structure is shown in Fig. 3(b). To implement a Golay sequence pair with Alamouti coding as the TS [7], we sent four sequence blocks in the X-polarization before the information payload; each block contained two guard intervals (17 symbols each) and TS (64 symbols) to make the duration of two blocks to match exactly the delay of the polarization multiplexer. Blocks $\mathrm{A}$ and $\mathrm{C}$ are Golay pairs: the spectra of blocks B and D were set to conj(FFT(C)) and conj(FFT(A)), respectively. As shown in the constellation diagram in Fig. 3(b), the information payload used 64-QAM, while the TS used QPSK with scaling to the four highest amplitude points of 64-QAM (red points) to achieve the best training signal to noise ratio. The training block was repeated after every 10,000 information symbols for polarization tracking. After accounting for $20 \%$ FEC overhead, the net spectral efficiency is $9.804 \mathrm{~b} / \mathrm{s} / \mathrm{Hz}$. After transmitting through $800 \mathrm{~km}$ with an EDFA every $80 \mathrm{~km}$, the received signals were first band-pass filtered by a waveshaper (WS), and then fed into an optical hybrid followed by balanced receivers. The transmitter laser and local oscillator were both external cavity lasers with $100-\mathrm{kHz}$ linewidths, and the LO was tuned to within $100 \mathrm{MHz}$ of the transmitter. The signals were captured by a 40-Gsamples/s real-time digital oscilloscope for offline processing. 

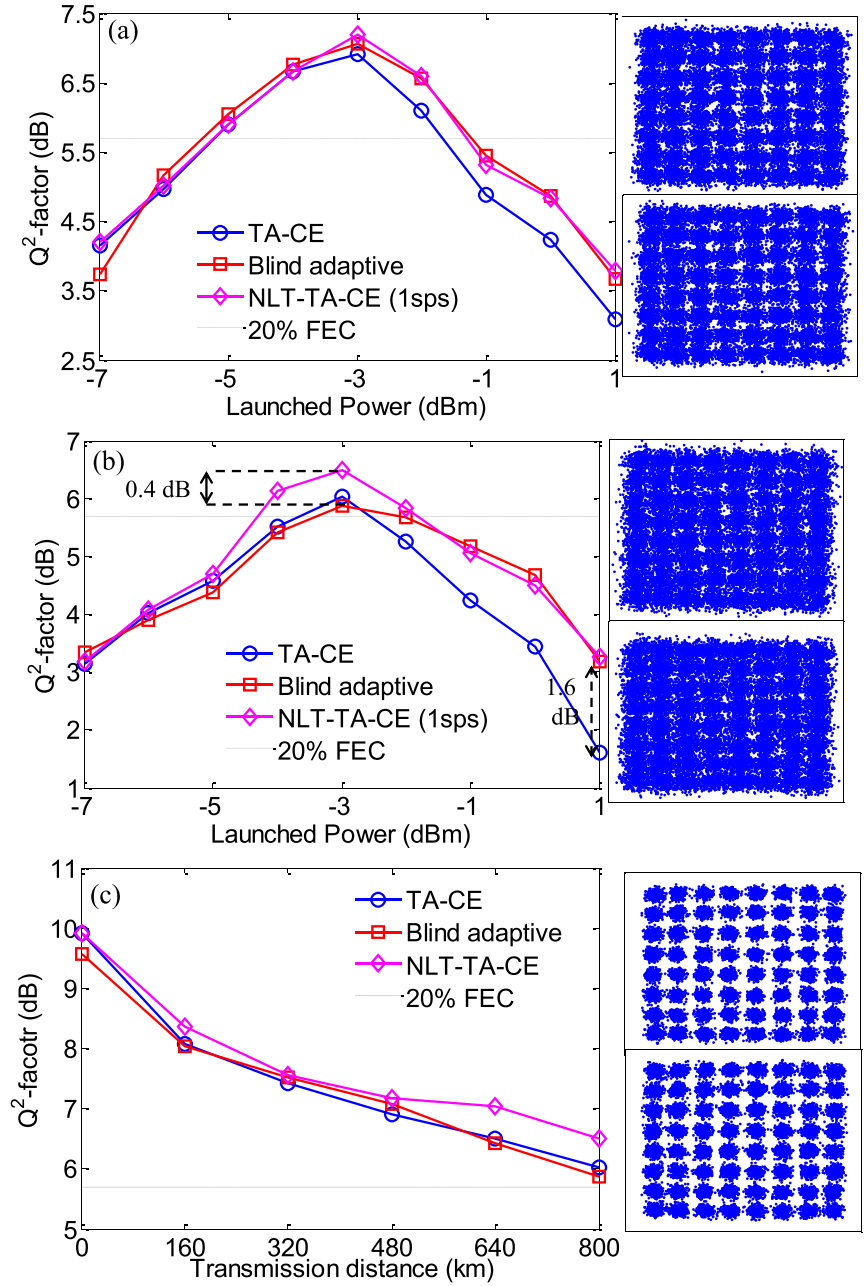

Fig. 4. Measured $\mathrm{Q}^{2}$-factors versus: (a) launched power for $480-\mathrm{km}$ transmission; (b) launched power for 800-km transmission; and (c) different transmission distances.

For the receiver's DSP, frequency offset compensation and frame synchronization were conducted first. Then, for the two training-aided systems, the same $\mathrm{CE}$ approach as in the simulations was performed, and then a low-complexity polyphase $2 \times 2$ MIMO frequency-domain equalizer [5] was used to remove all linear impairments. The DSP scheme of blind equalization system is the same as in simulation.

Figures 4(a) and (b) show the measured results with different launched powers for $480-\mathrm{km}$ and $800-\mathrm{km}$ transmission distances, respectively. Similar to the simulation results, TA-CE performs much worse than the blind scheme in the fiber nonlinearity limited region. NLT-TA-CE improves the nonlinearity tolerance to become comparable to the blind system, resulting in a $0.4-\mathrm{dB} Q^{2}$-factor increase after $800-\mathrm{km}$ transmission at the optimal launch power of $-3 \mathrm{dBm}$ and a $1.6 \mathrm{~dB}$ $Q^{2}$-factor increase at a $1 \mathrm{dBm}$ launched power compared with TA-CE. The same received data sets were used for performance evaluation of different equalization systems, and the $\mathrm{Q}^{2}$ difference purely comes from the linear equalization performances. $Q^{2}$ versus transmission distance is plotted in Fig. 4(c), with the results taken at the optimal launched power for each distance. The TA-CE and blind equalization systems perform similarly, while the performance enhancement due to
NLT-TA-CE increases with distance. The system can achieve error-free transmission after $800 \mathrm{~km}$ assuming 20\% soft decision forward error correction (SD-FEC) with a 5.7-dB $Q^{2}$ requirement [16]. The six constellation diagrams show equalized signals (from top to bottom for both polarizations) with: NLT-TA-CE at $-3 \mathrm{dBm}$ launched power at $480-\mathrm{km}$; NLT-TA-CE at $-3 \mathrm{dBm}$ launched power at $800-\mathrm{km}$; and back to back transmission.

\section{Conclusions}

We have demonstrated that applying DBP nonlinearity compensation to just the TS provides a worthwhile improvement in system performance at a very low computational cost. For PDM-64QAM transmitted over $800 \mathrm{~km}$, the improvement in peak $Q^{2}$ was $0.4-\mathrm{dB}$.

\section{REFERENCES}

[1] P. J. Winzer, "Beyond 100G Ethernet," IEEE Commun. Mag., vol. 48, no. 7, pp. 26-30, Jul. 2010.

[2] T. Kobayash, A. Sano, A. Matsuura, Y. Miyamoto, and K. Ishihara, "Nonlinear tolerant spectral-efficient transmission using PDM 64-QAM single carrier FDM with digital pilot tone," J. Lightw. Technol., vol. 30, no. 24, pp. 3805-3815, Dec. 15, 2012.

[3] X. Zhou et al., "High spectral efficiency $400 \mathrm{~Gb} / \mathrm{s}$ transmission using PDM time-domain hybrid 32-64 QAM and training-assisted carrier recovery," J. Lightw. Technol., vol. 31, no. 7, pp. 999-1005, Apr. 1, 2013.

[4] W.-R. Peng, T. Tsuritani, and I. Morita, "Transmission of highbaud PDM-64QAM signals," J. Lightw. Technol., vol. 31, no. 13, pp. 2146-2162, Jul. 1, 2013.

[5] C. Zhu, A. V. Tran, F. N. Hauske, S. Chen, T. Anderson, and E. Skafidas, "Low-complexity fractionally-spaced frequency domain equalization with improved channel estimation for long-haul coherent optical systems," in Proc. OFC/NFOEC, Anaheim, CA, USA, 2013, pp. 1-3, paper OW4B.5.

[6] R. Elschner et al., "Experimental demonstration of a format-flexible single-carrier coherent receiver using data-aided digital signal processing," Opt. Exp., vol. 20, no. 27, pp. 28786-28791, Dec. 2012.

[7] A. V. Tran et al., " $8 \times 40-\mathrm{Gb} / \mathrm{s}$ optical coherent pol-mux single carrier system with frequency domain equalization and training sequences," IEEE Photon. Technol. Lett., vol. 24, no. 11, pp. 885-887, Jun. 1, 2012.

[8] C. C. Do et al., "Data-aided chromatic dispersion estimation for polarization multiplexed optical systems," IEEE Photon. J., vol. 4, no. 5, pp. 2037-2049, Oct. 2012.

[9] C. Zhu, L. B. Du, A. V. Tran, A. J. Lowery, and E. Skafidas, "Trainingaided PDM 64-QAM transmission with enhanced fiber nonlinearity tolerance," in Proc. Optical Fiber Communication Conf., San Francisco, CA, USA, 2014, paper M2A.7.

[10] E. Ip, "Nonlinear compensation using backpropagation for polarizationmultiplexed transmission," J. Lightw. Technol., vol. 28, no. 6, pp. 939-951, Mar. 15, 2010.

[11] L. B. Du and A. J. Lowery, "Improved single channel backpropagation for intra-channel fiber nonlinearity compensation in longhaul optical communication systems," Opt. Exp., vol. 18, no. 16, pp. 17075-17088, Jul. 2010.

[12] P. Poggiolini, "The GN model of non-linear propagation in uncompensated coherent optical systems," J. Lightw. Technol., vol. 30, no. 24, pp. 3857-3879, Dec. 15, 2012.

[13] S. Savory, "Digital filters for coherent optical receivers," Opt. Exp., vol. 16, no. 2, pp. 804-817, Jan. 2008

[14] C. Zhu et al., "Frequency-domain blind equalization for longhaul coherent pol-mux 16-QAM system with CD prediction and dual-mode adaptive algorithm," IEEE Photon. J., vol. 4, no. 5, pp. 1654-1661, Oct. 2012.

[15] S. Zhang, P.-Y. Kam, C. Yu, and J. Chen, "Decision-aided carrier phase estimation for coherent optical communications," J. Lightw. Technol., vol. 28, no. 11, pp. 1597-1607, Jun. 1, 2010.

[16] D. Chang et al., "LDPC convolutional codes using layered decoding algorithm for high speed coherent optical transmission," in Proc. Signal Processing Photonics Communications, 2012, pp. 1-3, paper OM1H.4. 